\title{
Surface Plasmon Resonance Based Fiber Optic Sensor: Theoretical Simulation and Experimental Realization.
}

\author{
${ }^{1}$ Murtadha Faaiz Sultan ${ }^{1}$, Ali A. Al-Zuky ${ }^{1}$ and ${ }^{2}$ Shehab A. Kadhim \\ ${ }^{1}$ Department of Physics, Collage of Science, Al-Mustanasiyah University, Baghdad-Iraq. \\ ${ }^{2}$ Laser \& Optoelectronics Research Center, Ministry of Science \& Technology, Baghdad-Iraq. \\ *Corresponding author: murtadhaphysics@yahoo.com.
}

\begin{abstract}
Surface plasmon resonance (SPR) based fiber optic sensor with two types of plasmonic metals (gold and silver) are theoretically studied and implemented experimentally. Performance characteristics like sensitivity, signal to noise ratio, the figure of merit, and resolution are evaluated for both simulated and fabricated sensor. The results of theoretical and experimental studies and also between the sensor with the gold layer and that with silver have been compared. Sensitivity is larger for the sensor with the gold layer while the signal to noise ratio and figure of merit are larger for the sensor with the silver layer for both the experimental and theoretical studies. The resolution is slightly larger for the sensor with the gold layer and there is some difference between the resolution of the simulated and that of the fabricated sensor. [DOI: 10.22401/JUNS.21.1.11]
\end{abstract}

Keywords: surface plasmon resonance, fiber optic sensor, performance characteristics.

\section{Introduction}

Many developments occur in the field of sensing in several areas of the science, like biology, biomedical, biochemistry, chemistry, and physics. These developments presented solutions to several problems associated with the sensors. The problems which can confront the sensors associated with the sensor size, its ability to sense small variations, the cost, the amount of the sample to be sense, and its performance parameters like sensitivity, resolution, signal to noise ratio... etc $[1,2]$.

Surface plasmon resonance (SPR) has accomplished many achievements to provide advanced sensors especially after its participation with the technology of optical fiber. This type of sensors, SPR-based fiber optic sensor, now is benefit in many fields due to its significant advantages like extremely fast response, very small amount of sample, label free samples, miniature components, monitoring in hard-to-reach environments, real-time analysis, remote sensing, low coast as well as its good performance parameters [3-5]. Surface plasmons are an excitations appear due to the oscillations of free electrons at the interface between metal and dielectric. These excitations are TM polarized waves that propagate along the interface. The field correlated with these excitations is exponentially decays in both metal and dielectric media. Due to the transverse polarization of magnetic field of these waves, surface plasmons can be excited using p-polarized light incident on the metal surface. When the propagation constant of the incident light equal to that of the surface plasmon wave, the resonance take place and resulting in the energy transfer from incident light to surface plasmon wave. The propagation constant of surface plasmon wave depends on the dielectric constant of the exterior medium in contact with the metal. Any change in the dielectric constant leads to change in the propagation constant of the surface plasmon wave and hence change in the propagation constant of the incident p-polarized light at resonance. The change determination of the propagation constant of the incident light at resonance, leads to determination of the dielectric constant of the exterior medium. In order to excite the surface plasmons, an optical elements like high refractive index prism, diffraction grating, optical fiber are used [6,7].

\section{Theoretical simulation}

The sensing of the SPR sensors is based on attenuated total reflection (ATR) with Kretschmann configuration. This type of sensors mainly consists of three layers: optical fiber core, thin layer of metal, and sensing medium as shown in Fig.(1). Small portion of optical fiber unclad firstly and then a metal layer deposit directly on the core. According to 
spectral interrogation method a polychromatic light is launched into one of the ends of the optical fiber and the transmitted light at the other end is detected using optical spectrum analyzer (OSA). At a specific wavelength a resonance occur and at this point a sharp dip in the transmitted power appears. Any change of the refractive index of the sensing medium, which is adjacent to the metal surface, leads to shifting in the resonance wavelength [8].

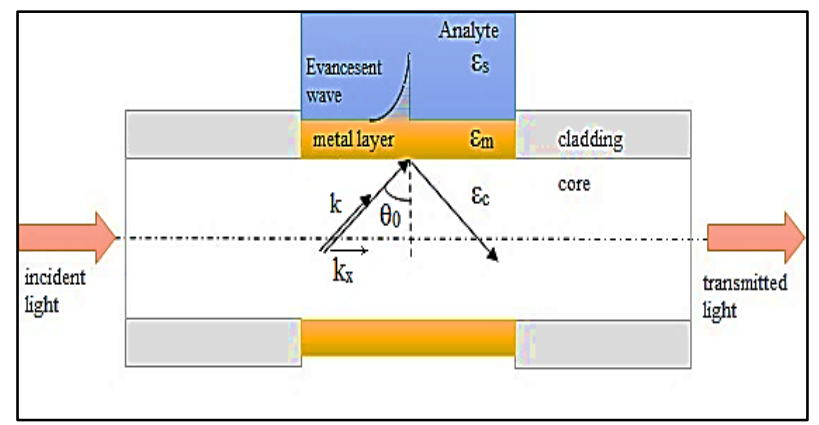

Fig.(1): Illustration diagram of three layers system: the silica core of fiber, the sensitive metal layer and the medium to be analyzed [8].

\subsection{Optical fiber core}

The first layer used to fabricate SPR based fiber optic sensor is the fiber core which is mainly made from fused silica. The dispersion relation, which describe the relation between the refractive index and the wavelength, of fused silica is given by [9]:

$n_{c}(\lambda)=C_{0}+C_{1} \lambda^{2}+C_{2} \lambda^{4}+\frac{C_{3}}{\left(\lambda^{2}-a\right)}+\frac{C_{4}}{\left(\lambda^{2}-a\right)^{2}}+$ $\frac{C_{5}}{\left(\lambda^{2}-a\right)^{3}}$

Where $C_{0}, C_{1}, C_{2}, C_{3}, C_{4}, C_{5}$ and $a$ are numerical values given as:

$$
\begin{array}{ll}
C_{0}=1.4508554 & C_{1}=-0.0031268 \\
C_{2}=-38.1 \times 10^{-6} & C_{3}=0.0030270 \\
C_{4}=-77.9 \times 10-6 & C_{5}=1.8 \times 10-6
\end{array}
$$$$
a=0.035 \text {. }
$$

\subsection{Plasmonic metal}

According to Drude's theory, the dielectric constant of any metal is given by [10]:

$\varepsilon_{m}=1-\frac{\lambda^{2} \lambda_{c}}{\lambda_{p}^{2}\left(\lambda_{c}+i \lambda\right)}$

where $\lambda_{c}$ and $\lambda_{p}$ are the collision and the plasma wavelengths of the metal respectively and given for gold and silver in Table (1).
Table (1)

The collision and the plasma wavelengths of gold and silver [11, 12].

\begin{tabular}{|c||c||c|}
\hline metal & $\begin{array}{c}\text { Plasma } \\
\text { wavelength } \\
\lambda_{\boldsymbol{p}}(\boldsymbol{m})\end{array}$ & $\begin{array}{c}\text { Collision } \\
\text { wavelength } \lambda_{\boldsymbol{c}} \\
(\boldsymbol{m})\end{array}$ \\
\hline \hline Gold $(\mathrm{Au})$ & $1.6826 \times 10^{-7}$ & $8.9342 \times 10^{-6}$ \\
\hline \hline Silver $(\mathrm{Ag})$ & $1.4541 \times 10^{-7}$ & $17.614 \times 10^{-6}$ \\
\hline
\end{tabular}

\subsection{Sensing medium}

The medium that adjacent to the surface of plasmonic metal is called the sensing medium. Different concentrations of a solution of glycerin in water have different refractive indices so that it can be considered as a sensing medium. The dielectric constant of sensing medium is $\boldsymbol{\varepsilon}_{\mathrm{s}}$ and if $\mathrm{n}_{\mathrm{s}}$ is the refractive index of sensing medium then $\boldsymbol{\varepsilon}_{\mathrm{s}}=\mathrm{n}_{\mathrm{s}}{ }^{2}$. To get an excitation of surface plasmon wave, a condition of resonance should be satisfied. The resonance condition is given by [13]:

$\frac{2 \pi}{\lambda} n_{1} \sin \theta=\operatorname{Re}\left[k_{s p}\right]$

where $\mathrm{n}_{1}$ is core refractive index, $\theta$ is the incident angle on the metal-core interface, and $R e\left[k_{s p}\right]$ is a real part of the surface plasmon wave vector that can written as [13]:

$k_{s p}=\frac{\omega}{c} \sqrt{\frac{\varepsilon_{m} \varepsilon_{s}}{\varepsilon_{m}+\varepsilon_{s}}}=\frac{2 \pi}{\lambda} \sqrt{\frac{\varepsilon_{m} n_{s}^{2}}{\varepsilon_{m}+n_{s}^{2}}}$

where $\varepsilon_{\mathrm{m}}$ and $\varepsilon_{\mathrm{s}}$ are the dielectric constants of metal and sensing medium respectively and $\omega$ is the frequency of the incident wave while $c$ is a speed of light.

\subsection{Transmitted power}

N-layer model offer a calculation of the reflection coefficients by using matrix method.

From these coefficients, the power of the light transmitted in the optical fiber are calculated. By taking into account the two types of polarization, $-\mathrm{P}$ and $-\mathrm{S}$, the transmitted light power will be calculated separately with the reflection coefficients for the $-s$ and $-p$ polarizations. The total transmitted power will be deducted from the average of the contributions of the two polarizations:

$$
P_{\text {trans }}=\frac{P_{p}+P_{s}}{2}
$$

Then the total transmitted power is [14]: 
$P_{\text {trans }}=\frac{1}{2}\left[\frac{\int_{\theta_{c r}}^{\pi / 2} R_{p}^{N_{r e f}(\theta)} \frac{n_{1}^{2} \sin \theta \cos \theta}{\left(1-n_{1}^{2} \cos ^{2} \theta\right)^{2}} d \theta}{\int_{\theta_{c r}}^{\pi / 2} \frac{n_{1}^{2} \sin \theta \cos \theta}{\left(1-n_{1}^{2} \cos ^{2} \theta\right)^{2}} d \theta}+\frac{\int_{\theta_{c r}}^{\pi / 2} R_{s}^{N_{r}} e^{(\theta)} \frac{n_{1}^{2} \sin \theta \cos \theta}{\left(1-n_{1}^{2} \cos ^{2} \theta\right)^{2}} d \theta}{\int_{\theta_{c r}}^{\pi / 2} \frac{n_{1}^{2} \sin \theta \cos \theta}{\left(1-n_{1}^{2} \cos ^{2} \theta\right)^{2}} d \theta}\right]$

Since that the SPR phenomenon can occur only for a p-polarization light, so that the final expression of $P_{\text {trans }}$ can be given by:

$P_{\text {trans }}=\frac{1}{2}\left[\frac{\int_{\theta_{c r}}^{\pi / 2} R_{p}^{N_{r e f}(\theta)} \frac{n_{1}^{2} \sin \theta \cos \theta}{\left(1-n_{1}^{2} \cos ^{2} \theta\right)^{2}} d \theta}{\int_{\theta_{c r}}^{\pi / 2} \frac{n_{1}^{2} \sin \theta \cos \theta}{\left(1-n_{1}^{2} \cos ^{2} \theta\right)^{2}} d \theta}+1\right] \ldots .$.

where $R_{p}$ and $R_{s}$ are the reflectivity of the light of polarization -p and -s respectively and $N_{\text {ref }}$ is the number of reflections accomplished by a beam making an angle $\theta$ with the normal to the core/metal interface in the sensing region and it written as [15]:

$N_{\text {ref }}(\theta)=\frac{L}{D \tan \theta}$

$L$ and $D$ are the length of the sensing region and the optical fiber core diameter respectively.

The critical angle of the core/clad interface is [15]:

$\theta_{c r}=\sin ^{-1}\left(\frac{n_{c l}}{n_{1}}\right)$

where $\mathrm{n}_{\mathrm{cl}}$ and $\mathrm{n}_{1}$ are the refractive indices of the clad and core of the optical fiber respectively.

\subsection{Performance characteristics}

Performance characteristics to be studied are: sensitivity, signal to noise ratio, figure of merit, and resolution. In the case of spectral interrogation, sensitivity can defined as the change in resonance wavelength per unit change in refractive index of the sensing medium and it can be written as [16]:

$S_{n}=\frac{\delta \lambda_{r e s}}{\delta n_{s}}$

Signal to noise ratio (SNR), figure of merit (FOM), are inversely proportional to the width of SPR spectral curve and can written as [16]:

$S N R=\frac{\delta \lambda_{\text {res }}}{\delta \lambda_{1 / 2}}$

$F O M=\frac{S_{n}}{\delta \lambda_{1 / 2}}$
Finally, the resolution of the sensor can be defined as the minimum of change in refractive index detectable by the sensor and given as [16]:

$R=\frac{\delta n_{s}}{\delta \lambda_{\text {res }}} \delta \lambda_{D R}$

where $\delta \lambda_{\mathrm{DR}}$ is the spectral resolution of the optical spectrometer analyzer used to measure the resonance wavelength and it considered to be equal to $0.01 \mu \mathrm{m}$.

\section{Experimental devices and setup}

The scheme illustrates the components of the SPR based fiber optic sensor setup is shown in Fig.(2).

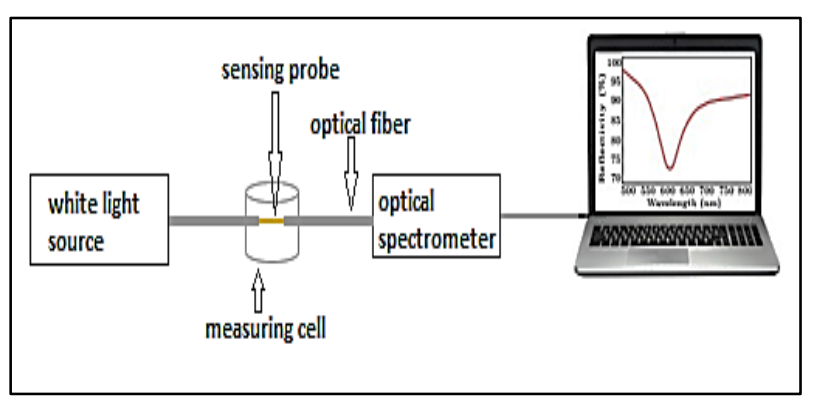

Fig.(2): Experimental setup of SPR fiber optic sensor.

This setup consists of a white light from halogen source, step index multimode optical fiber from Thorlab, measuring cell of about $3 \mathrm{ml}^{3}$ made from PVC (Polyvinyl Chloride) and finally the optical spectrum analyzer USB4000+UV-VIS-NIR from Ocean Optics which connected to a computer with help of software provided by Ocean Optics.

\subsection{Sensor probe fabrication}

A small portion $(10 \mathrm{~mm})$ of step index multimode optical fiber of core diameter $600 \mu \mathrm{m}$ and numerical aperture 0.24 is unclad firstly by a suitable blade. The unclad portion is cleaned with acetone and subsequently with methanol and distilled water. Then it is coated with about 50nm thickness of metal (gold and silver) by using the DC sputtering. The sputtering machine used was from MTI Company, USA. 


\section{2 preparation a solution of various refractive indices}

The sensitive zone of the sensor is immersed in different glycerin/water solutions with different concentrations and then different $n_{s}$ refractive indices. The refractive indices of the solutions have been measured by using (ZEISS 13743) refractometer. Fig.(3) illustrate the relation between the solution concentrations and the refractive index.

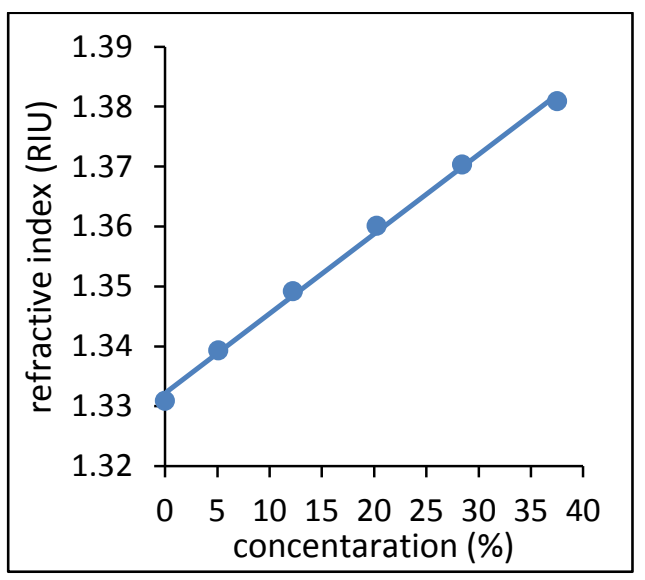

Fig.(3): Refractive index of glycerin solutions as a function of the solution concentration.

\subsection{Transmission-wavelength curve}

The spectra are obtained by recording the transmission curves $\mathrm{T}$ of the light through the optical fiber. The transmission $T$ is calculated from the ratio of the intensity $I$ measured in the presence of a sample (the sensing medium) and the intensity of the optical signal reference $I_{0}$ that measured in the absence of a sample. $T$, calculated as a percentage, and it is a function of the wavelength expressed in $\mathrm{nm}$. The $T$-wavelength curve is called SPR curve and at specific wavelength called resonance wavelength, a sharp dip occur in $T$. The position of this dip is depend on the refractive index of the sensing medium.

\section{Results and Discussion}

The values of the parameters used in the numerical calculations and also in the experimental study were: fiber optic numerical aperture $(\mathrm{NA})=0.22$, fiber core diameter (D) $=600 \mu \mathrm{m}$, sensing length $(\mathrm{L})=10 \mathrm{~mm}$, metal layer thickness $(\mathrm{d})=50 \mathrm{~nm}$ and different values $(1.33,1.34,1.35,1.36$, and 1.37) of refractive index from glycerin-water solutions.

Fig.(4) shows the SPR response curves for both simulated and fabricated sensor with gold and silver layer at different refractive indices of sensing medium. The resonance wavelength increase as refractive index of the sensing medium increase Fig.(5) for the sensor with both of gold and silver layer. This behavior can be justified by the resonance condition of surface plasmon wave given in equation 3 . If the refractive index of the sensing medium is large, then the real part of the propagation constant will be large and hence the resonance condition will satisfied at large value of wavelength. Also if the refractive index of the sensing medium is small, then the real part of the propagation constant will be small and hence the resonance condition will satisfied at small value of wavelength. It is clear that the width and the dip position of each SPR response curve is different for the sensor with gold layer than that for the sensor with silver at each refractive index and also the magnitude of shifting of the dip position as the refractive index increase. These differences make the performance parameters, which are depend on the SPR curve width, the position of dip, and the amount of the shifting, changing with the changing of plasmonic metal type. Table (2) shows the performance parameters for both simulated and fabricated sensor with the gold and silver layer. The sensor with gold layer has sensitivity larger than that with silver while the sensor with silver layer has SNR, FOM, and resolution greater than that of the sensor with gold layer. The results of the numerical simulation seams in good agreement with that of experimental realization study. 

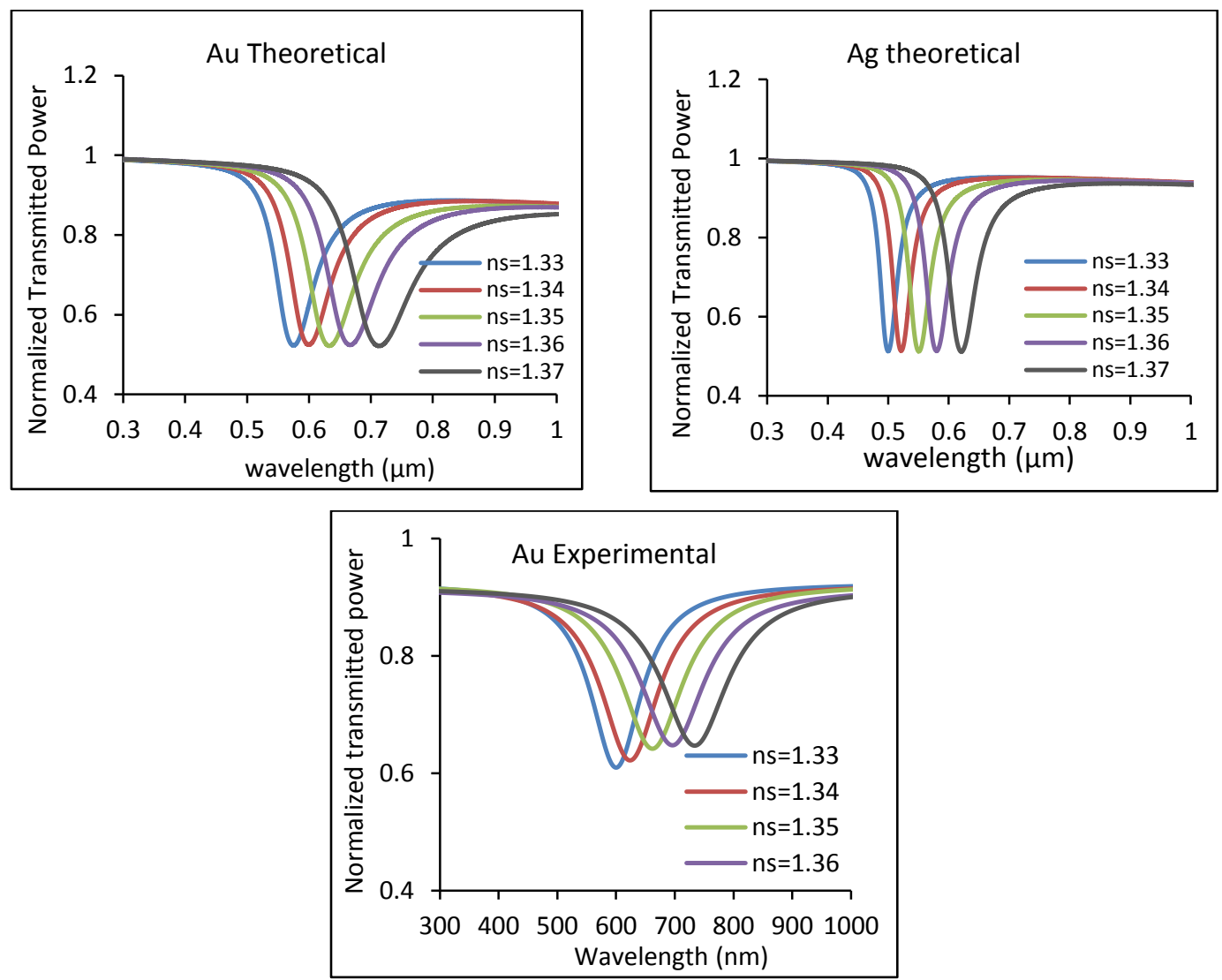

Fig.(4): Theoretical and experimental SPR response curves of the sensor with gold and silver layer of thickness 50nm at different refractive indices of sensing medium.

Table (2)

Theoretical and experimental performance parameters of the sensor with gold and silver.

\begin{tabular}{|c|c|c|c|c|c|c|c|c|}
\hline & \multicolumn{4}{|c|}{ Theoretical } & \multicolumn{4}{|c|}{ Experimental } \\
\hline metal & $\operatorname{Sn}(\mu m / R I U)$ & SNR & FOM & $R(R I U)$ & $\operatorname{Sn}(\mu m / R I U)$ & SNR & FOM & $R(R I U)$ \\
\hline gold & 3.43399 & 0.28783 & 32.04 & 0.000607 & 3.340 & 0.354 & 27.8 & 0.000459 \\
\hline silver & 3.00299 & 0.5944 & 67.20 & 0.000697 & 2.980 & 0.427 & 74 & 0.000434 \\
\hline
\end{tabular}
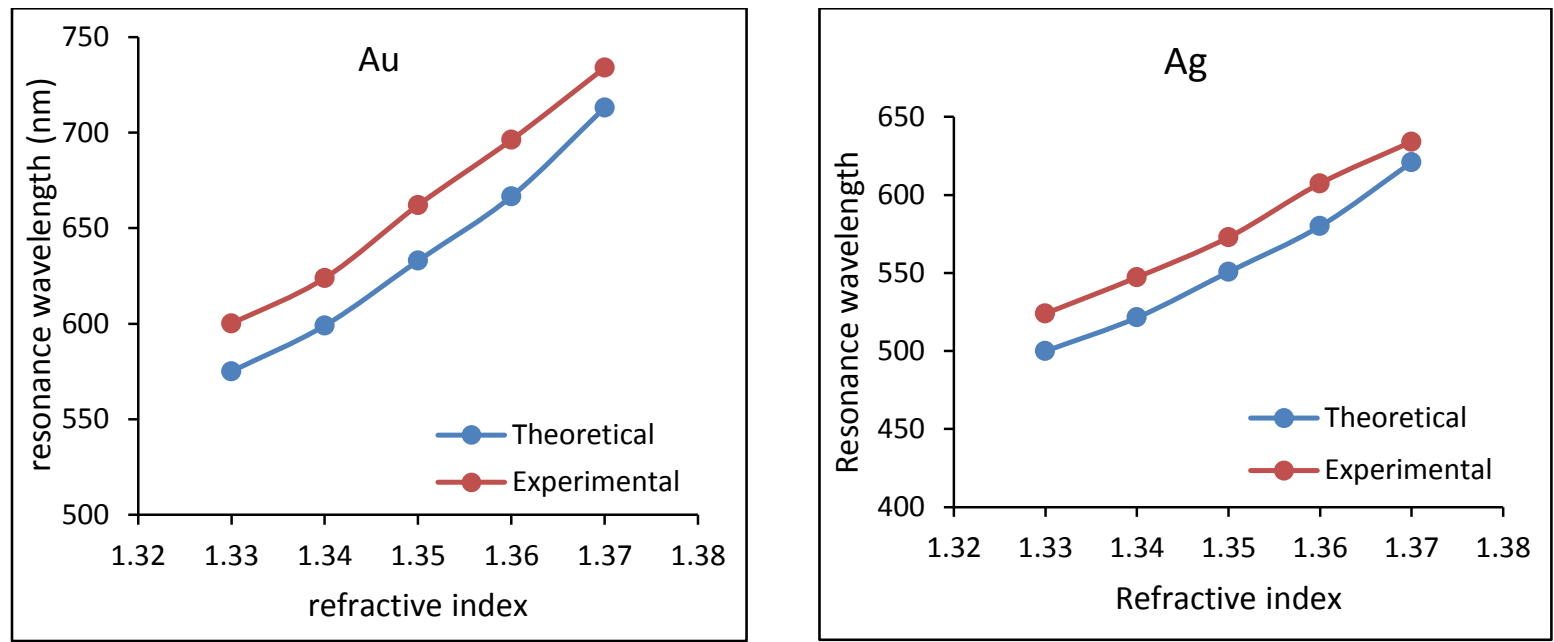

Fig.(5): Resonance wavelength as a function of refractive index for the sensor with gold and silver layer for both theoretical and experimental studies. 


\section{Conclusion}

Surface plasmon resonance (SPR) based fiber optic sensor is experimentally and numerically. The performance parameters different as the plasmonic metal type different. Sensitivity is greater with gold than that with silver while the other performance parameters are larger with the silver than that with gold. The N-layer model with matrix formalism showed a good method to simulate SPR fiber optic sensor and also to evaluate its performance parameters. In the other hand, the performance parameters and response curves of fabricated sensor show good agreement with that of simulated sensor.

\section{Refrences}

[1] Ligler F.S. and C.A.R. Taitt, "Optical biosensors: present and future", Gulf Professional Publishing, p.215, 2002.

[2] Homola, J., S.S. Yee, and G. Gauglitz, "Surface plasmon resonance sensors: review". Sensors and Actuators B: Chemical, 54(1): p. 3-15, 1999.

[3] Jorgenson, R. and S. Yee, "A fiber-optic chemical sensor based on surface plasmon resonance", Sensors and Actuators B: Chemical, 12(3): p. 213-220, 1993.

[4] Jorgenson, R.C., et al., "Multi-wavelength surface plasmon resonance as an optical sensor for characterizing the complex refractive indices of chemical samples", Sensors and Actuators B: Chemical, 14 (1-3): p. 721-722, 1993.

[5] Homola, J., "Optical fiber sensor based on surface plasmon excitation". Sensors and actuators B: chemical, 29(1-3): p. 401-405, 1995.

[6] Lin, W.B., et al., "Development of a fiberoptic sensor based on surface plasmon resonance on silver film for monitoring aqueous media", Sensors and Actuators B: Chemical, 75(3): p. 203-209, 2001.

[7] Srivastava, S.K. and B.D. Gupta, "Influence of ions on the surface plasmon resonance spectrum of a fiber optic refractive index sensor", Sensors and Actuators B: Chemical, 156(2): p. 559-562, 2011.

[8] Sharma, A.K. and B. Gupta, "Fibre optic sensor based on long-range surface plasmon resonance: a theoretical analysis",
Journal of Optics A: Pure and Applied Optics, 9(7): p. 682, 2007.

[9] Paek, U., G. Peterson, and A. Carnevale, "Dispersionless Single-Mode Lightguides With a Index Profiles". Bell System Technical Journal, 60(5): p. 583-598, 1981.

[10] Maier, S.A., Plasmonics: fundamentals and applications, Springer Science \& Business Media, p.211, 2007.

[11] Gupta, B.D., S.K. Srivastava, and R. Verma, "Fiber optic sensors based on plasmonics", World Scientific, p. 157, 2015.

[12] Ordal, M., et al., "Optical properties of the metals al, co, cu, au, fe, pb, ni, pd, pt, $a g$, $t i$, and $w$ in the infrared and far infrared", Applied optics, 22(7): p. 10991119, 1983.

[13] Shah, K., et al., "Theoretical study of surface plasmon resonance based fiber optic sensor utilizing an additional layer of zinc oxide", Optik-International Journal for Light and Electron Optics, 127(14): p. 5743-5749, 2016.

[14] Geddes, C., "Reviews in plasmonics", Springer New York, p.132, , 2010.

[15] Sharma, A.K. and B. Gupta, "Fiber optic sensor based on surface plasmon resonance with nanoparticle films", Photonics and Nanostructures-Fundamentals and Applications, 3(1): p. 30-37, 2005.

[16] Cennamo, N., et al., "Low cost sensors based on SPR in a plastic optical fiber for biosensor implementation", Sensors, 11(12): p. 11752-11760, 2011. 